\title{
Decade Wide Bandwidth Integrated Very Low Noise Amplifier
}

\author{
J.G. Bij de Vaate, E.E.M. Woestenburg, R.H. Witvers, R. Pantaleoni, \\ Netherlands Foundation for Research in Astronomy, Dwingeloo, The Netherlands \\ P.O. Box 2, 7990 AA Dwingeloo, \\ The Netherlands \\ Phone: +31-521-595100 Fax: $+31-521-597332$ \\ E-mail: vaate@nfra.nl
}

\section{Abstract}

This paper describes the design, realization and characterization of an integrated Low Noise Amplifier (LNA) with a bandwidth from 0.4 to $8 \mathrm{GHz}$. A very good noise figure is achieved in this frequency band. The design uses a source impedance of 150 $\mathrm{ohm}$ in the contrary to the standard of $50 \mathrm{ohm}$. The load impedance is $50 \mathrm{ohm}$.

The use of a non-50 input source impedance creates new possibilities for the design of the power and noise matching circuitry. A combination of a good power and noise match can be achieved for a very broad band, with a minimum of components. This concept requires a non-standard antenna (source) output impedance $(150 \mathrm{ohm})$, which is for most antenna principles easier to realize then $50 \mathrm{ohm} \mathrm{[1].}$

The LNA will be part of an active antenna phased array system, which should operate from 0.2 to $2 \mathrm{GHz}$ [2].

\section{Design structure}

Figure 1 gives the schematic of the two stage amplifier. The first stage uses source inductive feedback [3,4], which moves the input impedance (S11) of the FET closer to the optimum noise match condition. A minimum noise figure can then be achieved in combination with a good input return loss of the amplifier. Due to the selected input impedance of $150 \mathrm{ohm}$, a minimum of matching circuitry is required in the first stage. A small integrated inductor in series with the gate is sufficient. The integration of this inductor on the IC does not degrade the performance, which would be the case for a large inductor because of a relatively high series resistance of integrated inductors. The intrinsic gain roll-off in the transistor is not compensated in the matching of the first stage but in the frequency dependent feedback loop of the second stage. The inductor in the loop provides a gain increase in this stage for the higher frequencies.

In Figure 2 the simulations of the LNA are plotted. From 0.4 to $8 \mathrm{GHz}$ a gain of more then $17 \mathrm{~dB}$ and a noise figure below $1.2 \mathrm{~dB}$ is combined with a good input and output return-loss. 


\section{Results}

The LNA has been fabricated by Philips Microwave Limeil in their 0.2um PsHEMT GaAs process. Figure 3 shows a photograph of the IC.

Figure 4 gives the measurements performed on a wafer prober in combination with the simulation results in a $50 \mathrm{ohm}$ environment. The simulations and the measurements correspond very well.

However, the full characterization of the LNA cannot be performed on the wafer prober by using a standard noise figure meter. For the source impedance of $150 \mathrm{ohm}$, probes, cables but especially a calibrated noise source are not available. Instead a hot/cold source measurement method has been used with a $150 \mathrm{ohm}$ resistor at two different temperatures. Figure 5 is a photograph of the test board on which the bare IC was mounted for the hot/cold noise measurements. On the input of the IC a long 150 $\mathrm{ohm}$ line connects the noise source, a $150 \mathrm{ohm}$ resistor. The long line ensures temperature stability of the DUT when the resistor is cooled down. The temperature stability of the DUT was verified with a temperature sensor on the board (the SO package on the photograph).

The noise power measured at the output of the LNA is given by the following formula:

$P n=k B G_{T}\left(T_{e}+T_{\text {noisesource }}\right)$,

where $G_{T}$ represents the gain of the LNA, $T_{\text {noisesource }}$ the temperature of the $150 \mathrm{ohm}$ load which is attached to the input of the amplifier and $T_{e}$ the effective noise temperature of the LNA. Pn has been measured for a noise source temperature of 295 Kelvin and 78 Kelvin, where 78 Kelvin is the temperature of liquid nitrogen. The Y factor, defined by $\mathrm{P}_{\text {nhot }} / \mathrm{P}_{\text {ncold }}$, can then be calculated. The $\mathrm{T}_{\mathrm{e}}$ follows with (2).

$T_{e}=\frac{T_{h o t}-Y T_{\text {cold }}}{Y-1}$

The calculated Noise temperature has to be corrected for the loss of the microstrip connection line. The result of the (corrected) measurement is given in figure 6 , where the noise figure is plotted in $\mathrm{dB}$ according to (3).

$$
N F(d B)=10^{* 10} \log \left(T_{e} / 290+1\right)
$$

The difference between the simulated and the measured noise figure is smaller then $0.2 \mathrm{~dB}$. An extension of the measurements to cover the complete relevant bandwidth still has to be performed.

\section{Conclusion}

With the selection of a 'new' impedance standard, that is not equal to 50 (or 75) ohm, a significant performance improvement is achieved with a low noise amplifier design. The LNA is very suitable for, but not limited to, the use in active antenna designs for receive-only systems. Providing the availability of e.g. $150 \mathrm{ohm}$ duplex filters, other telecom systems can benefit as well. This is especially the case for CMOS receivers since the transistor input impedance levels of a CMOS transistor are similar to the impedances of the PsHEMT. 


\section{References}

[1] Woestenburg E.E.M. et.al., 'Wide Band Low Noise Active Antenna for Radio Astronomical Arrays', Antennas\&Propagation, 2000 Davos

[2] Smolders A.B. et.al., 'Dual Beam Wide-Band Beamformer with Integrated Antenna Array', Antennas\&Propagation, 2000 Davos

[3] Vendelin G.D., 'Feedback effects on the noise performance of GaAs FET's', IEEE MTT-S, 1975

[4] Williams D.R. et.al., 'L-band Cryogenically-Cooled GaAs FET Amplifier', Microwave Journal, Oct 1980

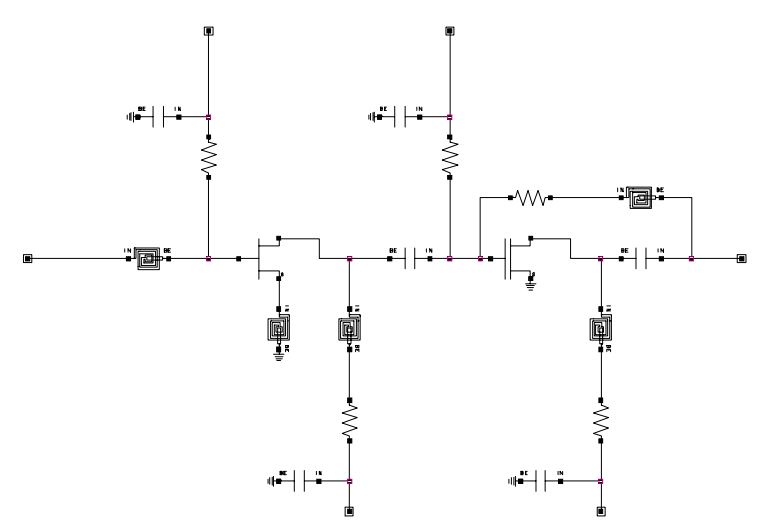

Figure 1. Schematic of the LNA

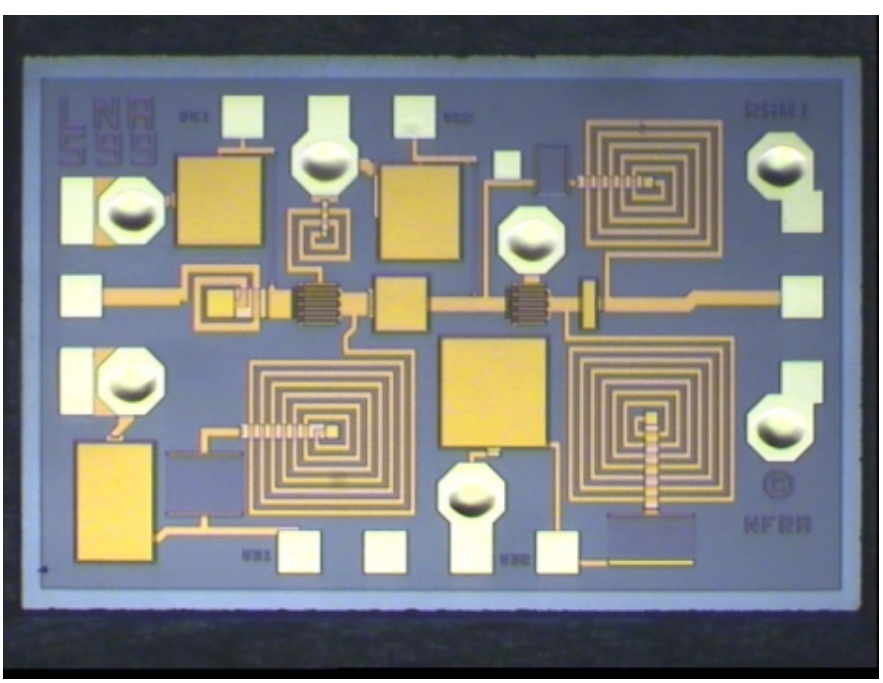

Figure 3. Photograph of the LNA IC.

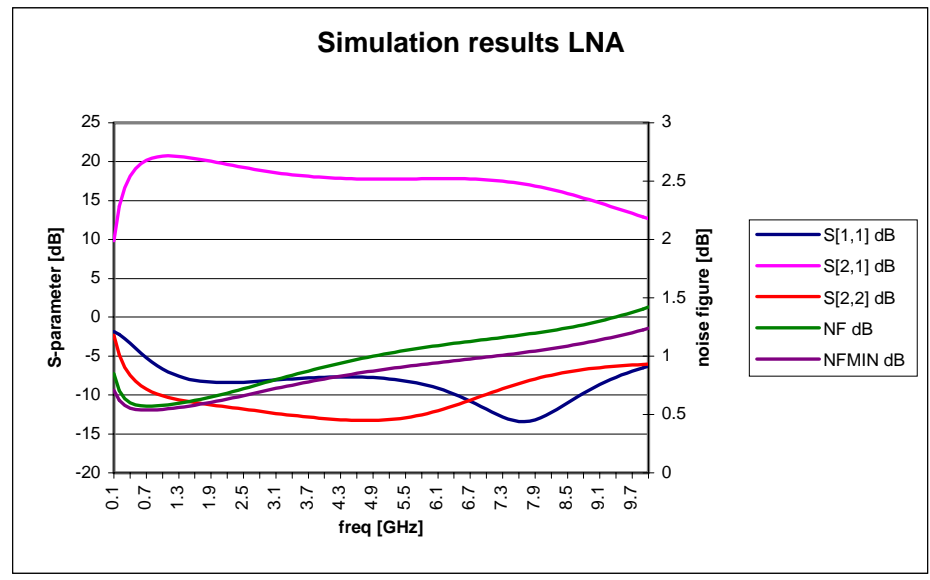

Figure 2 Simulations of the LNA

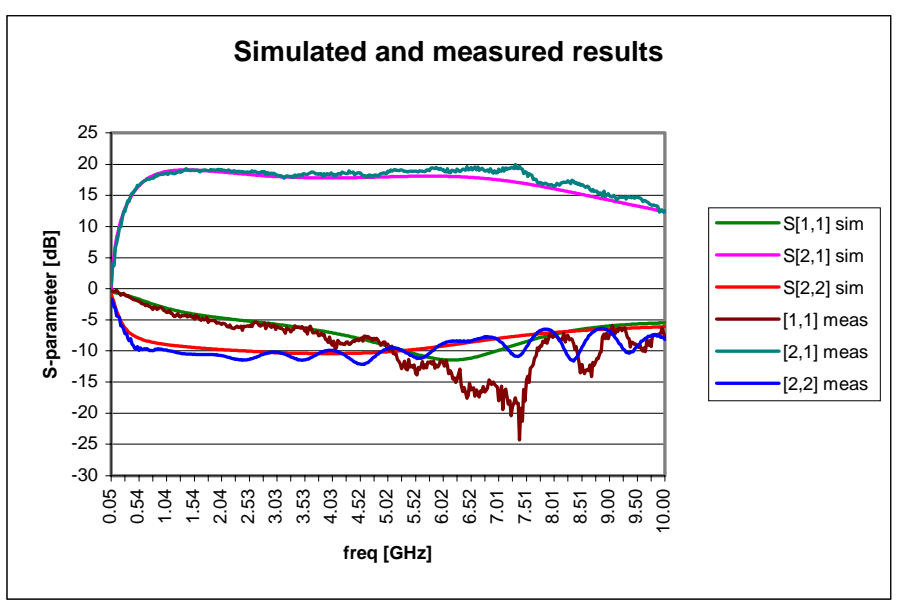

Figure 4. Simulated and measured results 


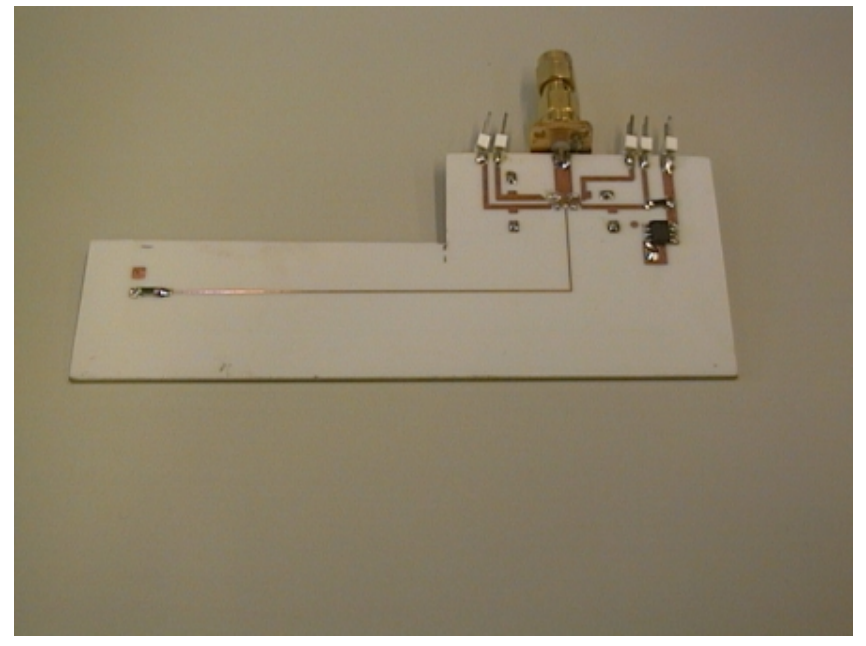

Figure 5. Photograph of the test board

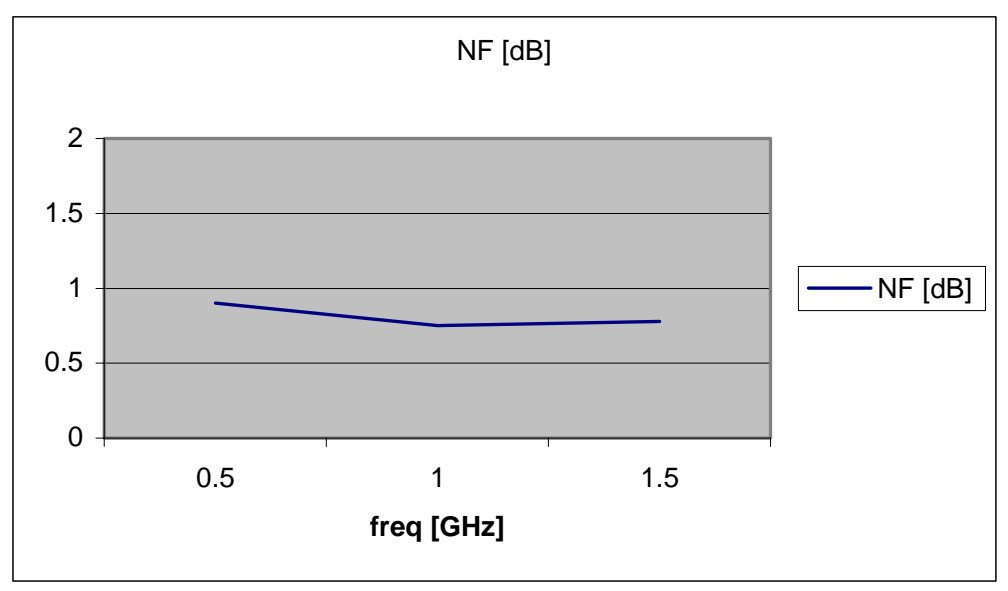

Figure 6. Measured noise figure with $150 \mathrm{ohm}$ noise source 